\title{
VASSILIEV INVARIANTS AND SIMILARITY OF KNOTS
}

\author{
YOSHIYUKI OHYAMA
}

(Communicated by Ronald Stern)

\begin{abstract}
We show that for any knot $K$ and any natural number $n$, we can construct infinitely many knots, all of whose finite type invariants of order at most $n$ coincide with those of $K$.
\end{abstract}

In 1990, Vassiliev [10] defined a sequence of knots invariants that are at least as powerful as all of the quantum group invariants of knots. After that Birman and Lin succeeded in giving an axiomatic description for Vassiliev invariants in [3].

On the other hand, the author [6] introduced the notion " $n$-trivial" for a knot diagram and Taniyama [9] generalized it to " $n$-similar". In this paper, we show the following theorem by making use of $(n+1)$-similar knots.

Main Theorem. For any knot $K$ and any natural number $n$, there exists infinitely many knots with the same Vassiliev invariant of order $m(1 \leq m \leq n)$ as that of $K$.

Our definition of Vassiliev invariants follows the Birman-Lin axioms in [3] or Bar-Natan [1]. Whenever we have a numerical invariant of knots, we can define an invariant of a knotted graph as

$$
v\left(K_{D}\right)=v\left(K_{+}\right)-v\left(K_{-}\right),
$$

where a knotted graph denotes an image of an immersion of a circle in $R^{3}$ whose only singularities are transverse double points and where $K_{D}, K_{+}$, and $K_{-}$denote the diagrams of knotted graphs which are identical except near one point, as in Figure 1.

An invariant $v$ is called a Vassiliev invariant of order $m$ and is denoted by $v_{m}$, if for any knotted graph $K^{n}$ with $n>m$ double points:

$$
v\left(K^{n}\right)=0 .
$$

An invariant of oriented knots in $R^{3}$ is called an invariant of finite type if it is a Vassiliev invariant of order $m$ for some $m \in N$. In [3], Birman and Lin show that after a suitable change of variables, each coefficient in the Taylor expansion of Jones, HOMFLY, and Kauffman polynomials is an invariant of

Received by the editors April 5, 1993.

1991 Mathematics Subject Classification. Primary 57M25.

Key words and phrases. Vassiliev invariant, $n$-trivial, $n$-similar. 


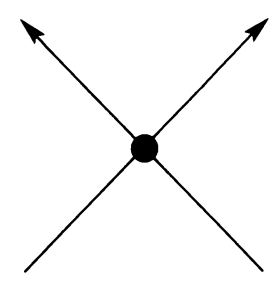

$K_{D}$

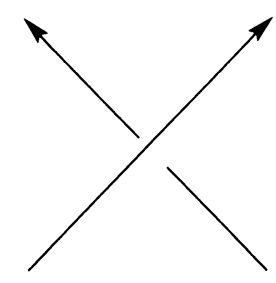

$K_{+}$

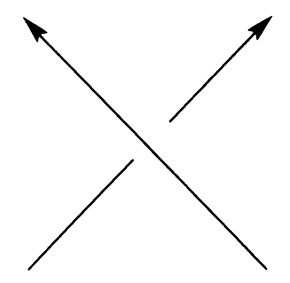

$K_{-}$

FIGURe 1

finite type. Moreover, the Vassiliev invariants include all of the quantum group invariants (see $[4,2])$.

Now we consider the similarity of knots. The author introduced the notion of an $n$-trivial knot and the triviality index in [6]. Taniyama [9] extended them to an $n$-similar knot to a given knot and the similarity index.

Let $K$ be a knot, and let $D(K)$ be a diagram for $K$. We are interested in very special diagrams. To explain their main property, let $C$ be the set of crossing points in $D(K)$ and let $n$ be a positive integer. Let $\mathscr{A}=\left\{A_{1}, A_{2}, \ldots, A_{n}\right\}$ be a collection of $n$ pairwise disjoint, nonempty, not necessarily proper subsets of $C$. Let $\mathscr{A}_{j_{1}, j_{2}, \ldots, j_{m}}$ denote the subset $\left\{A_{j_{1}}, A_{j_{2}}, \ldots, A_{j_{m}}\right\} \subset \mathscr{A}$. The property which is of interest to us is:

(*) There is a fixed knot type $L$ such that the diagram which is obtained from $D(K)$ by switching the crossings in $\mathscr{A}_{j_{1}, j_{2}, \ldots, j_{m}}$

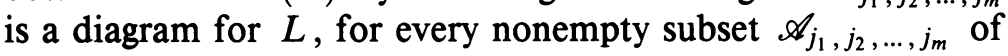
$\mathscr{A}$.

If this occurs, we say that $K$ is $n$-similar to $L$. In the special case when $L$ is the unknot, we say that $K$ is $n$-trivial.

One might wonder whether these definitions are vacuous. Do $n$-trivial knots exist? Theorem 1 (which was proved in an earlier paper [7] by the author and Ogushi) shows that there are many examples of $n$-trivial knots:

Theorem 1 [7]. For any natural number $n>1$, there are infinitely many knots which are $n$-trivial but not $(n+1)$-trivial.

Corollary 2. For any knot $L$ and any natural number $n$ there exist infinitely many composite knots which are $n$-similar to $L$, namely, the connected sum of $L$ with any $n$-trivial knot $K$.

From now on we calculate the Vassiliev invariant of order $m$ for a knot which is $n$-similar to $L$. Let $K$ be a knot that is $n$-similar to $L$ and $\widetilde{K}$ the diagram of $K$ with the collection of crossing points $\left\{A_{1}, A_{2}, \ldots, A_{n}\right\}$ which gives an $n$-similarity. Let $A_{i}=\left\{c_{i 1}, c_{i 2}, \ldots, c_{i \alpha(i)}\right\}$ and $\varepsilon_{i j}$ be the sign of $c_{i j}$ defined as in Figure 2.

By

$$
K\left(\begin{array}{cccc}
1 & 2 & \cdots & k \\
i_{1} & i_{2} & \cdots & i_{k}
\end{array}\right)
$$

we denote the knotted graph which is obtained from $K$ by switching the crossings at $c_{11}, c_{12}, \ldots, c_{1 i_{1}-1}, c_{21}, c_{22}, \ldots, c_{2 i_{2}-1}, \ldots, c_{k 1} c_{k 2}, \ldots, c_{k i_{k}-1}$ and collapsing crossing points to double points at $c_{1 i_{1}}, c_{2 i_{2}}, \ldots, c_{k i_{k}}$. 


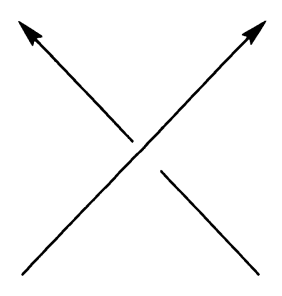

$+1$

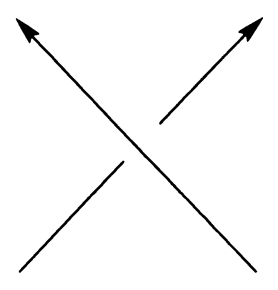

$-1$

FIGURE 2

Lemma 3. If a knot $K$ is n-similar to a knot $L$, then the Vassiliev invariant of order $m$ of $K$ is of the following form,

$$
v_{m}(K)=v_{m}(L)+\sum_{\substack{1 \leq i_{j} \leq \alpha(j) \\
j=1,2, \ldots, n}} \varepsilon_{1 i_{1}} \varepsilon_{2 i_{2}} \cdots \varepsilon_{n i_{n}} v_{m}\left(K\left(\begin{array}{cccc}
1 & 2 & \cdots & n \\
i_{1} & i_{2} & \cdots & i_{n}
\end{array}\right)\right)
$$

Proof (the techniques are similar to those used in [6]). In the case $n=1$, by using (1) inductively, we have

$$
v_{m}(K)=v_{m}\left(K\left(\begin{array}{c}
1 \\
\infty
\end{array}\right)\right)+\sum_{1 \leq i_{1} \leq \alpha(1)} \varepsilon_{1 i_{1}} v_{m}\left(K\left(\begin{array}{c}
1 \\
i_{1}
\end{array}\right)\right),
$$

where $K\left(\begin{array}{c}1 \\ \infty\end{array}\right)$ denotes the knot obtained from $K$ by switching the crossings in $A_{1}$. Since a knot $K\left(\begin{array}{c}1 \\ \infty\end{array}\right)$ is the same type as $L$, we have the formula in Lemma 3.

We suppose that in the case $n=k-1$, Lemma 3 holds. If $K$ is $k$-similar to $L$ with respect to $\left\{A_{1}, A_{2}, \ldots, A_{k}\right\}, K$ is $(k-1)$-similar to $L$ whose $(k-1)$-similarity is given by $\left\{A_{1}, A_{2}, \ldots, A_{k-1}\right\}$. Then

$$
v_{m}(K)=v_{m}(L)+\sum_{\substack{1 \leq i_{j} \leq \alpha(j) \\
j=1,2, \ldots, k-1}} \varepsilon_{1 i_{1}} \varepsilon_{2 i_{2}} \cdots \varepsilon_{k-1 i_{k-1}} v_{m}\left(K\left(\begin{array}{cccc}
1 & 2 & \cdots & k-1 \\
i_{1} & i_{2} & \cdots & i_{k-1}
\end{array}\right)\right)
$$

Here we calculate

$$
v_{m}\left(K\left(\begin{array}{cccc}
1 & 2 & \cdots & k-1 \\
i_{1} & i_{2} & \cdots & i_{k-1}
\end{array}\right)\right)
$$

by using $A_{k}$ in the same way as we did in the case $n=1$. It follows that $v_{m}(K)=v_{m}(L)$

$$
\begin{aligned}
& +\sum_{\substack{1 \leq i_{j} \leq \alpha(j) \\
j=1,2, \ldots, k-1}} \varepsilon_{1 i_{1}} \varepsilon_{2 i_{2}} \cdots \varepsilon_{k-1 i_{k-1}} v_{m}\left(K\left(\begin{array}{ccccc}
1 & 2 & \cdots & k-1 & k \\
i_{1} & i_{2} & \cdots & i_{k-1} & \infty
\end{array}\right)\right) \\
& +\sum_{\substack{1 \leq i_{j} \leq \alpha(j) \\
j=1,2, \ldots, k}} \varepsilon_{1 i_{1}} \varepsilon_{2 i_{2}} \cdots \varepsilon_{k i_{k}} v_{m}\left(K\left(\begin{array}{cccc}
1 & 2 & \cdots & k \\
i_{1} & i_{2} & \cdots & i_{k}
\end{array}\right)\right) .
\end{aligned}
$$

To prove Lemma 3, it is enough to show

$$
\sum_{\substack{1 \leq i_{j} \leq \alpha(j) \\
j=1,2, \ldots, k-1}} \varepsilon_{1 i_{1}} \varepsilon_{2 i_{2}} \cdots \varepsilon_{k-1 i_{k-1}} v_{m}\left(K\left(\begin{array}{ccccc}
1 & 2 & \cdots & k-1 & k \\
i_{1} & i_{2} & \cdots & i_{k-1} & \infty
\end{array}\right)\right)=0 .
$$


To obtain the above formula, we consider the knot $K\left(\begin{array}{l}k \\ \infty\end{array}\right)$ that is the same type as $L$ and $(k-1)$-similar to $L$. By the induction hypothesis,

$$
\begin{aligned}
v_{m}\left(K\left(\begin{array}{c}
k \\
\infty
\end{array}\right)\right)=v_{m}(L)+\sum_{\substack{1 \leq i_{j} \leq \alpha(j) \\
j=1,2, \ldots, k-1}} \varepsilon_{1 i_{1}} \varepsilon_{2 i_{2}} \cdots \varepsilon_{k-1 i_{k-1}} \\
\times v_{m}\left(K\left(\begin{array}{ccccc}
1 & 2 & \cdots & k-1 & k \\
i_{1} & i_{2} & \cdots & i_{k-1} & \infty
\end{array}\right)\right) .
\end{aligned}
$$

Since $v_{m}\left(K\left(\begin{array}{c}k \\ \infty\end{array}\right)\right)=v_{m}(L)$, we have

$$
\sum_{\substack{1 \leq i_{j} \leq \alpha(j) \\
j=1,2, \cdots, k-1}} \varepsilon_{1 i_{1}} \varepsilon_{2 i_{2}} \cdots \varepsilon_{k-1 i_{k-1}} v_{m}\left(K\left(\begin{array}{ccccc}
1 & 2 & \cdots & k-1 & k \\
i_{1} & i_{2} & \cdots & i_{k-1} & \infty
\end{array}\right)\right)=0 .
$$

This completes the proof of Lemma 3 .

In Lemma 3,

$$
K\left(\begin{array}{cccc}
1 & 2 & \cdots & n \\
i_{1} & i_{2} & \cdots & i_{n}
\end{array}\right)
$$

has $n$ double points. Then

$$
v_{m}\left(K\left(\begin{array}{cccc}
1 & 2 & \cdots & n \\
i_{1} & i_{2} & \cdots & i_{n}
\end{array}\right)\right)=0 \quad \text { for } m<n
$$

by (2). Therefore, by considering a knot that is $(n+1)$-similar to $K$, the Main Theorem follows from Corollary 2 and Lemma 3.

Remark. The same result has been proved by Stanford [8] and also follows from the work of Lin (see [5, 2]). Lin proves that if $K$ is any knot and if $K(m)$ is its $m$ th iterated (untwisted) Whitehead double, then all Vassiliev invariants of order $\leq(m+1)$ of $K(m)$ are zero. On the other hand, Taniyama [9] shows that if a knot $K$ is $n$-trivial, then its untwisted double is $(n+1)$-trivial. Then Lin's result can be also obtained by using our techniques.

\section{ACKNOWLEDGMENT}

The author expresses his appreciation to the referee for valuable suggestions.

\section{REFERENCES}

1. D. Bar-Natan, On the Vassiliev invariants, preprint, Harvard Univ., 1992.

2. J. S. Birman, New points of view in knot theory, Bull. Amer. Math. Soc. (N.S.) 28 (1993), 253-287.

3. J. S. Birman and X.-S. Lin, Knot polynomials and Vassiliev invariants, Invent. Math. 111 (1993), 225-270.

4. X.-S. Lin, Vertex models, quantum groups and Vassiliev's knot invariants, preprint, Columbia Univ., 1991.

5. __ Finite type link invariants of 3-manifolds, preprint, Columbia Univ., 1992.

6. Y. Ohyama, A new numerical invariant of knots induced from their regular diagrams, Topology Appl. 37 (1990), 249-255.

7. Y. Ohyama and Y. Ogushi, On the triviality index of knots, Tokyo J. Math. 13 (1990), 179-190.

8. T. Stanford, Braid commutators and Vassiliev invariants, preprint, Columbia Univ., 1992. 
9. K. Taniyama, On similarity of links, Gakujutu Kenkyu (issued by the school of education of Waseda University) 14 (1992), 33-36.

10. V. A. Vassiliev, Cohomology of knots spaces, Theory of Singularities and its Applications (V. I. Arnold, ed.), Adv. Soviet Math., vol. 1, Amer. Math. Soc., Providence, RI, 1990, pp. 9-21.

Department of Mathematics, Nagoya Institute of Technology, Gokiso, Showa-ku, NAGOYA, 466, JAPAN

E-mail address: H44685a@nucc.cc.nagoya-u.ac.jp 